\title{
L-Band Temporal Coherence Assessment and Modeling Using Amplitude and Snow Depth over Interior Alaska
}

\author{
Yusuf Eshqi Molan ${ }^{1, *(\mathbb{D})}$, Jin-Woo Kim ${ }^{1}$ (D), Zhong Lu ${ }^{1}$ (i) and Piyush Agram ${ }^{2}$ \\ 1 Roy M. Huffington Department of Earth Sciences, Southern Methodist University, Dallas, TX 75205, USA; \\ jinwook@mail.smu.edu (J.-W.K.); zhonglu@mail.smu.edu (Z.L.) \\ 2 Jet Propulsion Laboratory, California Institute of Technology, Pasadena, CA 91109, USA; \\ piyush.agram@jpl.nasa.gov \\ * Correspondence: yeshqimolan@smu.edu; Tel.: +1-469-623-0639
}

Received: 6 December 2017; Accepted: 16 January 2018; Published: 20 January 2018

\begin{abstract}
Interferometric synthetic aperture radar (InSAR) provides the capability to detect surface deformation. Numerous processing approaches have been developed to improve InSAR results and overcome its limitations. Regardless of the processing methodology, however, temporal decorrelation is a major obstacle for all InSAR applications, especially over vegetated areas and dynamic environments, such as Interior Alaska. Temporal coherence is usually modeled as a univariate exponential function of temporal baseline. It has been, however, documented that temporal variations in surface backscattering due to the change in surface parameters, i.e., dielectric constant, roughness, and the geometry of scatterers, can result in gradual, seasonal, or sudden decorrelations and loss of InSAR coherence. The coherence models introduced so far have largely neglected the effect of the temporal change in backscattering on InSAR coherence. Here, we introduce a new temporal decorrelation model that considers changes in surface backscattering by utilizing the relative change in SAR intensity between two images as a proxy for the change in surface scattering parameters. The model also takes into account the decorrelation due to the change in snow depth between two images. Using the L-band Advanced Land Observation Satellite (ALOS-2) Phased Array type L-band Synthetic Aperture Radar (PALSAR-2) data, the model has been assessed over forested and shrub landscapes in Delta Junction, Interior Alaska. The model decreases the RMS error of temporal coherence estimation from 0.18 to 0.09 on average. The improvements made by the model have been statistically proved to be significant at the $99 \%$ confidence level. Additionally, the model shows that the coherence of forested areas are more prone to changes in backscattering than shrub landscape. The model is based on L-band data and may not be expanded to C-band or X-band InSAR observations.
\end{abstract}

Keywords: InSAR; temporal coherence modeling; L-band; Interior Alaska

\section{Introduction}

Interferometric synthetic aperture radar (InSAR) provides an all-weather, day-or-night capability to remotely sense $\mathrm{mm}$ to $\mathrm{cm}$ scale surface deformation with a high spatial resolution of tens of meters or better (e.g., [1-4]). InSAR has been successfully used to detect surface deformation due to various mechanisms, such as volcanism, subsidence, permafrost, and landslides [5-9]. So far numerous methods and approaches have been developed to improve InSAR performance. However, temporal decorrelation, regardless of the processing methodology, is one of the major obstacles for all InSAR applications, especially over vegetated areas. The main sources of the loss of coherence, i.e., decorrelation, are temporal decorrelation, spatial decorrelation, volume decorrelation, thermal 
decorrelation, and processing errors (e.g., $[10,11])$. Generally, InSAR coherence decreases with increasing spatial and temporal baselines between two images.

InSAR coherence is sensitive to the changes in surface backscattering, which is dominated by the surface dielectric constant and roughness on the scale of the radar wavelength [12-14]. It has been documented that temporal coherence can be influenced by temporal variations of surface backscattering due to changes in soil moisture, snow depth, surface roughness, and vegetation biomass [12-22]. Simard et al. [12] found precipitation events to be the main cause of temporal decorrelation using fully-polarimetric airborne L-band acquisitions over forested landscapes with up to nine-day temporal baselines. Additionally, they argued that correlation decreases with increasing canopy height regardless of forest type and polarization. Zwieback et al. [15] evaluated soil moisture effects on L-band InSAR and revealed that the phase difference between two SAR images increased with increasing soil moisture difference, whereas the coherence decreased at the same time. Zhang et al. [20], in a case study using C-band ERS SAR data, assessed the relationship between InSAR coherence and soil moisture and inferred that the relation between the two may satisfy an exponential distribution.

Although the effect of the changes in surface backscattering on InSAR coherence has been documented (e.g., $[12,16,18])$, it has been largely neglected in the coherence models introduced so far. Temporal coherence, in general, is modeled as a univariate exponential function of the temporal baseline [10] with the assumption that the change in the position of scatterers, i.e., mutual displacements of scatterers, is the source of decorrelation [23]. However, we argue that other variables, in addition to the temporal baseline, should be added to the coherence function to compensate for the effect of the temporal variation of surface backscattering on InSAR coherence. In this paper, using ALOS-2 PALSAR-2 images, we analyzed the temporal decorrelation of forested and shrub landscapes in Delta Junction, Alaska, and introduced a new InSAR coherence model, which takes into account the effects of the temporal variations of surface backscattering on InSAR coherence. The model considers the changes in the geometry and dielectric constant of scatterers to be the main sources of decorrelations. The effect of the gradual and natural change in scatterers' geometry has been modeled as a decaying exponential function, which is equivalent to the exponential function of temporal coherence found in the literature $[10,13]$. The effect of the change in the surface backscattering, mainly due to the change in the dielectric constant of scatterers on InSAR coherence, has been modeled by utilizing the change in InSAR intensity as a proxy for it. The model also takes into account the decorrelation due to the change in snow depth between two images, which induces reversible and seasonal decorrelations. The model, in general, and with different constants, is applicable to model L-band InSAR coherence in other environments and may not be expanded to X-band or C-band SAR observations.

The importance of temporal decorrelation models and their practical use can be better understood by considering the following reasons. Basically, temporal changes of surface parameters describe processes occurring on time scales of the orbit repeat time. In other words, modeling temporal decorrelations provides a means to understand and remotely estimate a wide variety of surficial processes, such as vegetation growth, permafrost freezing and thawing, and soil moisture and vegetation layer induced effects [12].

For instance, it has been shown that both phase and coherence can be used to retrieve soil moisture (e.g., [15]). The coherence, being generally independent of deformation, provides a better means to estimate soil moisture. However, to retrieve soil moisture using temporal coherence, a decorrelation model should be implemented to separate the soil moisture-induced decorrelation, i.e., the change in the dielectric constant, from other decorrelation contributions, such as the decorrelation due to the change in the geometry of scatterers.

The second area of interest is in the design of orbit repeat for new satellite missions, which is driven by considering some important factors, such as tolerable error levels, the attainable baseline, and the expected decorrelation with the time of signals from the regions of interest to be mapped [12]. In this case, temporal decorrelation models can facilitate a priori assessment of the expected coherence levels of interferograms for a new satellite mission designed for a specific application. 
Finally, temporal decorrelation models can help better estimate vegetation layer parameters. The total InSAR coherence is the product of spatial, temporal, thermal, volume, and processing coherences (e.g., [23]). Most models used to invert vegetation layer parameters (in PolInSAR studies) only consider the volume decorrelation contribution of the interferometric coherence and ignore other decorrelation contributions. However, leaving non-volumetric decorrelations uncompensated leads to a less accurate parameter estimation. In repeat-pass InSAR systems, the most critical non-volumetric decorrelation contribution is the temporal decorrelation caused by the change of the geometric and/or dielectric properties of the scatterers [23] and its contribution to decorrelation can be quantified using temporal decorrelation models.

The rest of this paper is organized as follows: in Section 2, InSAR coherence estimates are presented over the study area, Delta Junction, Alaska, the temporal decorrelation model and evaluation with real data are described in Section 3, followed by discussions and conclusions in Sections 4 and 5, respectively.

\section{Study Area and Data}

Our test site, illustrated in Figure 1, is located in Delta Junction, interior Alaska. The area is mostly covered by forest and shrub landscapes [24] and underlain by dis-continuous permafrost. The Alaskan interior between the Alaska and Brooks Mountain Ranges has a strong continental climate with moderate temperatures and precipitation in summer and exceedingly cold and dry weather in winter [25]. The average minimum and maximum annual temperatures in Big Delta station (1937-2005), which is located in the study area, are $-6.9{ }^{\circ} \mathrm{C}$ and $-2.7^{\circ} \mathrm{C}$, respectively. The lowest and highest temperatures occur in January $\left(-23.7^{\circ} \mathrm{C}\right)$ and July $\left(20.8^{\circ} \mathrm{C}\right)$. Average total precipitation, average total snow-fall, and average snow depth are, respectively, about 29, 111.25, and 10.2 centimeters (National Weather Service (http:/ / www.wrcc.dri.edu)).

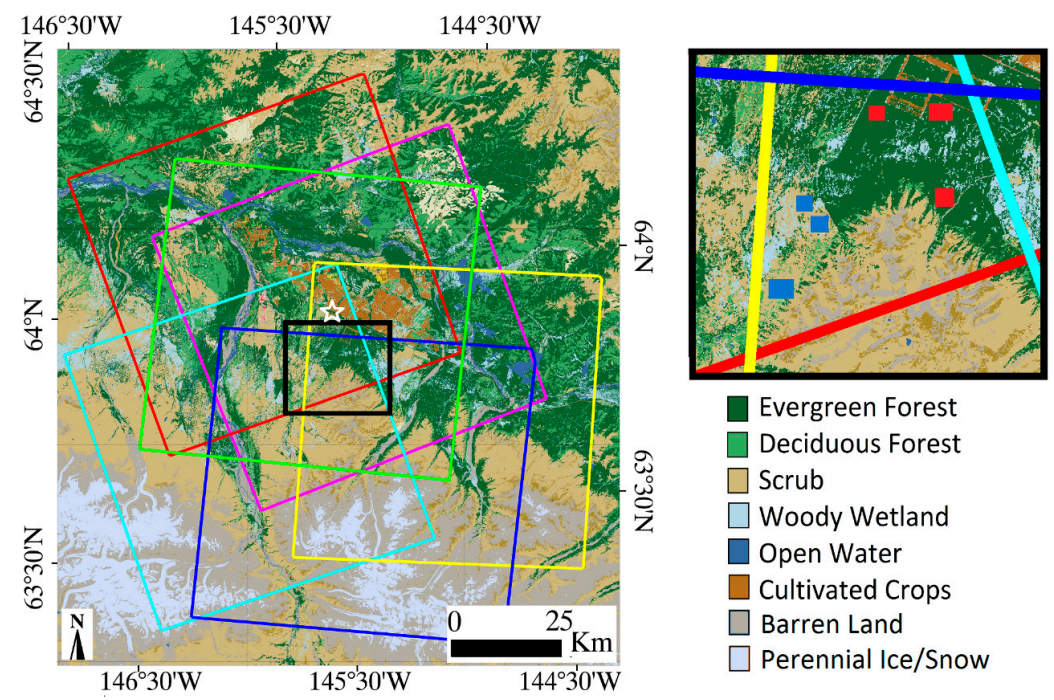

Figure 1. Land cover map of the study area (National Land Cover Database 2011 (NLCD 2011)) [24]. The orbit- frames covering the study area are shown with different colors (explained in Table 1). The black rectangle box shows the overlapping area. The forested and shrub patches are boxed in red and blue, respectively. The location of Snow Telemetry (SNOTEL) site, Granite Creek (963) (Natural Water and Climate Center (https:/ / wcc.sc.egov.usda.gov)), is shown by a white star.

To study the temporal evolution of InSAR coherence, 32 single look complex (SLC) SAR images of L-band ALOS-2 (23.6 cm wavelength) from three ascending and three descending orbital paths in the fine beam and horizontal-horizontal $(\mathrm{HH})$ polarization mode have been used. The data span from August 2014 to March 2017. Each group of the SLC SAR images have been co-registered based on a single master image, which optimizes the geometric and temporal coherence of the interferogram stack. 
The SLC images were then used to generate interferograms with a pixel size of about $30 \mathrm{~m} \times 30 \mathrm{~m}$. After removing topographic phase, simulated using the National Elevation Dataset (NED) DEM, and applying range spectral shift and azimuth common band filters, and a linear weighting window size of $5 \times 5$ (in pixels) was used to estimate correlation. Then, interferograms affected by ionospheric artifacts were excluded and a total number of 75 interferograms with no or very limited effects of ionospheric artifacts have been selected to analyze temporal coherence. Table 1 gives the information of the data and interferograms used in this study.

Table 1. Data used in this study. The letters A and D denote ascending and descending, respectively.

\begin{tabular}{cccc}
\hline Path-Frame & Orbit Direction & Number of Interferograms & Color of Frame on Figure 1 \\
\hline $0040-2330$ & D & 4 & Yellow \\
$0041-2330$ & D & 16 & Blue \\
$0042-2320$ & D & 10 & Green \\
$0137-1280$ & A & 10 & Magenta \\
$0138-1280$ & A & 29 & Red \\
$0139-1270$ & A & 6 & Cyan \\
\hline
\end{tabular}

For each of the two major land cover types in the study area, forest and shrub, three patches within flat areas with a total number of 1963 and 1729 pixels, respectively, on geo-referenced coherence images that are fully overlapped with all ALOS-2 observations have been selected. Figure 1 shows the selected patches in red (forest) and blue (shrub) boxes. For each of the interferograms, average coherence values of the selected pixels of each of the land cover types have been calculated. Therefore, each interferogram has two coherence values, one for each of the land cover types, evergreen forest and shrub. Figure 2 illustrates the scatter plot of the average coherence versus temporal baseline for the selected patches.
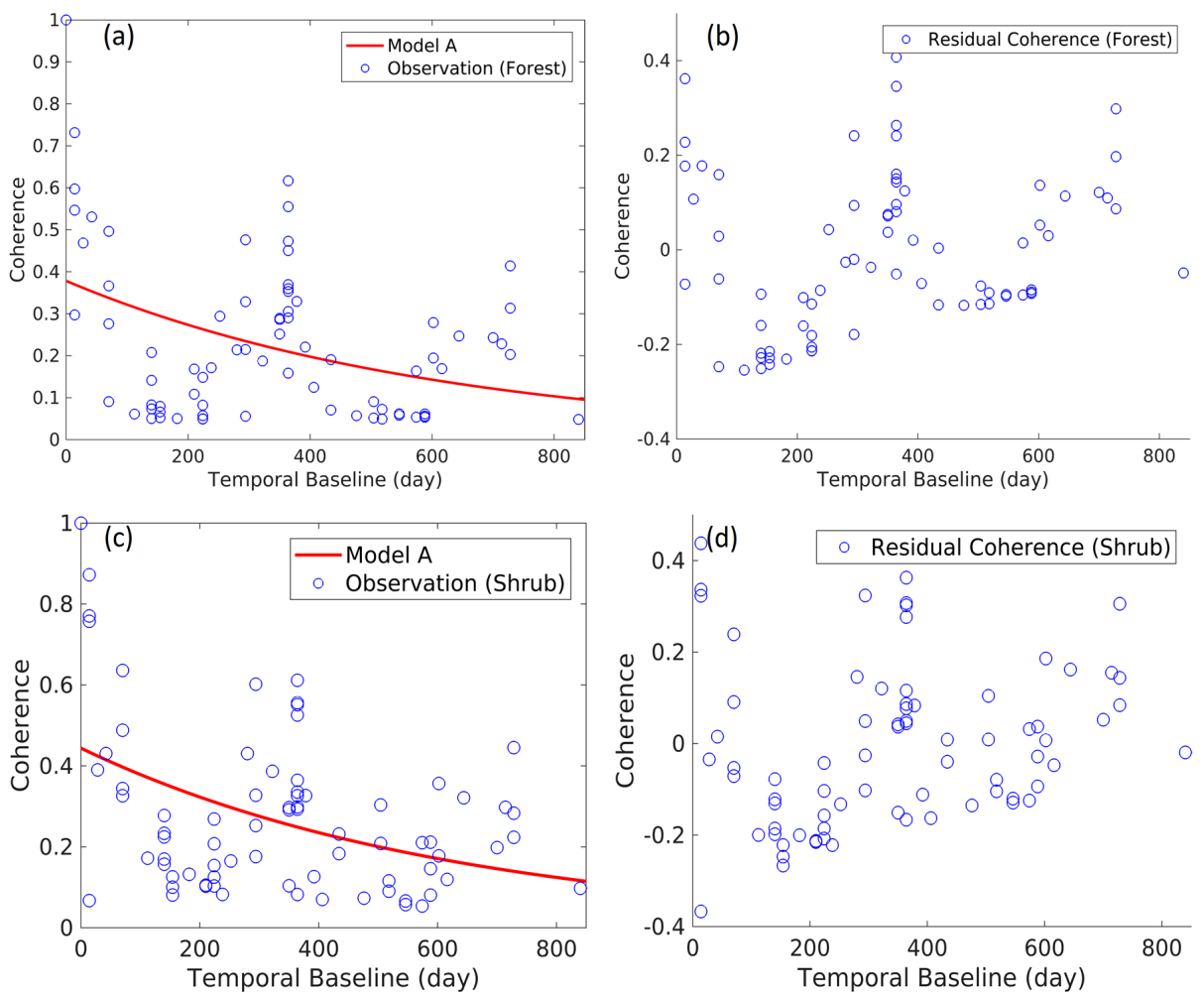

Figure 2. Scatter plots of observations and model A for forested (a) and shrub (c) land cover types. Scatter plots of residual coherences (observation-model A) for forested (b) and shrub (d) land cover types. 


\section{Methods}

\subsection{InSAR Coherence}

An InSAR coherence estimation image is a cross-correlation product of two co-registered complex-valued SAR images (e.g., [10,26,27]) which quantifies radar wavelength-scale changes in backscattering characteristics. Decorrelation, i.e., loss of coherence, is generally increased by increasing spatial and temporal baselines between two image acquisitions $[10,27,28]$. InSAR coherence assesses the accuracy of the estimated deformation and depends on the amount of phase error in an interferogram [28-30]. Over a small window of pixels, InSAR coherence is estimated by:

$$
\gamma=\left|\frac{\sum C_{1} C_{2}^{*} e^{-j \varnothing}}{\sqrt{\sum\left|C_{1}\right|^{2} \sum\left|C_{2}\right|^{2}}}\right|
$$

where $C_{1}$ and $C_{2}$ are complex-valued backscattering coefficients, $C_{2}{ }^{*}$ is the complex conjugate of $C_{2}, \varnothing$ is the deterministic phase due to baseline error, topography, or large deformation in the correlation window.

The total InSAR coherence is the product of spatial $\left(\gamma_{\text {spatial }}\right)$, temporal $\left(\gamma_{\text {temporal }}\right)$, thermal $\left(\gamma_{\text {thermal }}\right)$, volume $\left(\gamma_{\text {volume }}\right)$, and processing $\left(\gamma_{\text {processing }}\right)$ coherences $[4,10,28]$ :

$$
\gamma=\gamma_{\text {spatial }} \times \gamma_{\text {volume }} \times \gamma_{\text {temporal }} \times \gamma_{\text {thermal }} \times \gamma_{\text {processing }}
$$

The spatial (perpendicular) baselines of our dataset, except for two interferograms with spatial baselines of 308 and $347 \mathrm{~m}$, are smaller than $284 \mathrm{~m}$ with a mean of $\sim 108 \mathrm{~m}$, whereas the critical baseline of the data is about $11 \mathrm{~km}$. Therefore, a perpendicular baseline of $108 \mathrm{~m}$, i.e., the mean value of the perpendicular baselines, will decrease the coherence by the value of $\sim 0.01$ which is negligible. In long wavelength (L-band) SAR sensors, such as ALOS-2 PALSAR-2, the small perpendicular baseline will not affect the variation of spatial decorrelation much. Therefore, we assumed that the spatial decorrelation from the small range of change in the perpendicular baseline is constant. Additionally, with such small perpendicular baselines, the volumetric decorrelation is negligible (e.g., [12]). Here, we focus only on temporal decorrelation by assuming that other decorrelation terms are constant or relatively not significant.

\subsection{Temporal Coherence Modeling}

The temporal coherence is usually considered as a univariate exponential function of time (e.g., $[13,31])$ by taking the random motion of scatterers in the resolution cell to be the main source of decorrelation:

$$
\gamma_{A}=\gamma_{0} e^{-\frac{t}{\tau}}
$$

where subscript A denotes model A, $\gamma_{0}$ is initial coherence, $t$ is the time separation between two SAR images, and $1 / \tau$ is its decorrelation rate and is mainly dependent on the wavelength of the radar. Based on model A, the exponential decay of coherence values is expected in general by increasing the temporal baselines. However, the scatter plots of the observed coherence versus temporal baseline and the scatter plots of the residual coherence, i.e., observation-model A, versus the temporal baseline, illustrated in Figure 2, feature strong undulation with local peaks at temporal baselines around one and two years. Model A takes into account decorrelations due to long-term variations of the scatterers' geometry. In the real world, however, in addition to the natural and gradual long-term changes in the scatterers' geometry, seasonal and/or sudden changes in surface backscattering parameters may also contribute to temporal decorrelation. Generally, backscattering is dominated by the surface dielectric constant and roughness among other surface characteristics. Surface parameters, such as soil moisture, vegetation, and temperature alter dielectric constant and roughness and, consequently, backscattering 
coefficients (e.g., [17,22,32]). Therefore, other term(s) should be added to the temporal coherence function to compensate the effects of changes in surface backscattering between two image acquisitions.

In this paper, we have modified the coherence model to accommodate decorrelations due to the change in surface backscattering parameters. Since the SAR backscatter coefficient and, consequently, SAR intensity, varies as a function of the changes in surface parameters (e.g., [19,33]), here, we use the change in SAR intensity as a proxy for the changes in surface backscattering. Figure 3 shows a semi-logarithmic scatter plot of the coherence ratio, i.e., observation/model A, versus the relative intensity change between two images. Relative intensity is calculated by $r=\left|10 \log \left(i_{2} / i_{1}\right)\right|$, which $i_{2}$ and $i_{1}$ are SAR intensities of the first and second images, respectively. Considering the linear trend fitted to the semi-logarithmic scatter plots (note $R^{2}$ value and very small $p$-value of the linear regressions), model $\mathrm{B}$ is postulated to be:

$$
\gamma_{B}=\gamma_{0} e^{-\left(\frac{t}{\tau}+\frac{r}{\rho}\right)}
$$

where $r$ is the relative change in SAR intensity, i.e., the backscattering baseline, and $1 / \rho$ is its decorrelation rate. For each of the fitted linear trend, $R^{2}$ and $p$-value of the regression are calculated and shown on the plots. Note that if the $p$-value of a $t$-test is smaller than the common alpha values of $0.1,0.05$, and 0.01 (the confidence level of 90,95, and 99\%, respectively), the null-hypothesis is rejected. This means that the additional term related to the relative change in SAR intensity is likely correlated with temporal correlation.
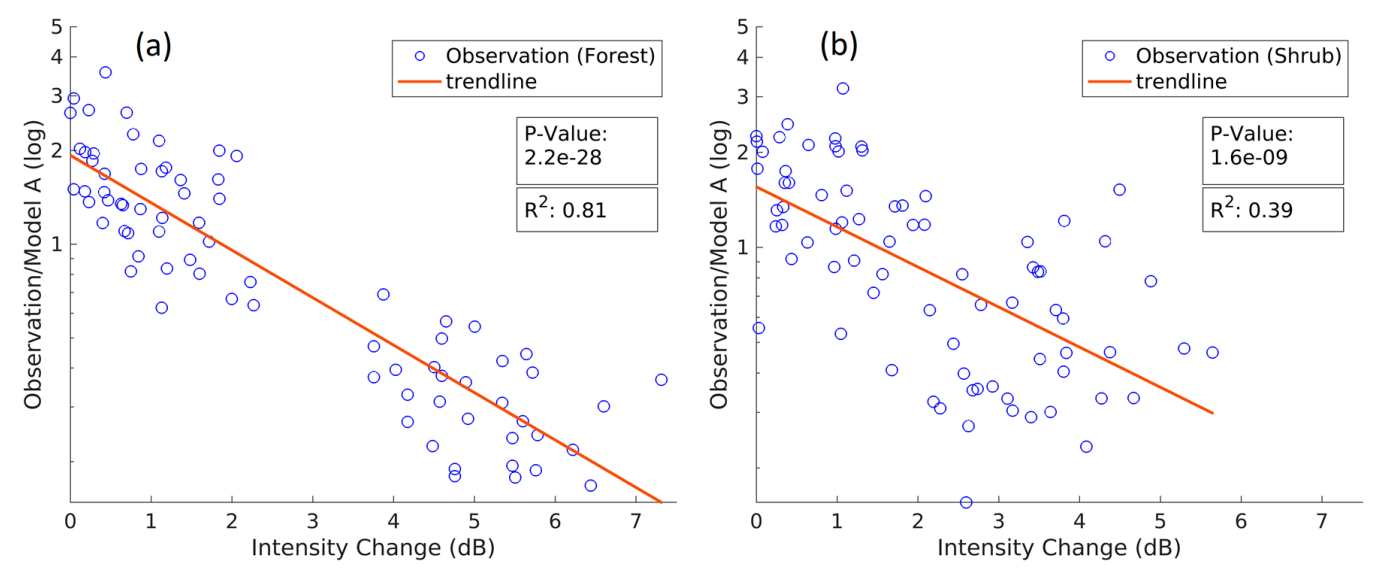

Figure 3. Semi-logarithmic scatter plot of coherence ratio, i.e., observation/model A, versus the relative intensity changes for forested (a) and shrub (b) land cover types.

The unknown parameters in models $\mathrm{A}$ and $\mathrm{B}$, i.e., $\gamma_{0}, \tau$, and $\rho$, can be estimated by solving the equations using known variables, i.e., $\gamma, t$, and $r$. The coherence, $\gamma$, is estimated using Equation (1). The temporal baseline of the interferograms, $t$, ranges between 14 and 840 days. Figure 4 illustrates the scatter plots of model B, observed coherence, and residual coherence values (observation-model B) for the two landscapes. For comparison, the scatter plot of water body's coherence is also shown in the figure. Additionally, Table 2 exhibits the model parameters and RMS error for each model. The RMS error values of model B for the both land cover types are smaller than those of model A, indicating that model B estimates more accurate coherence values. A detailed discussion of how significant the improvement is has been provided in Section 4.

Different snow depths between two images of an interferometric pair is one of the factors that can induce variations in surface scattering behavior, which, in turn, leads to decorrelation. Basically, between two winter images in stable frozen conditions with no change in soil moisture, the change in the dielectric constant is negligible and high coherence values can be expected for open areas [34]. However, the change in snow depth between the two images may change the surface scattering 
behavior, which, in turn, causes decorrelation. Here, we intend to modify our coherence model by adding the decorrelation term of snow depth changes. The basic assumption here is that the intensity and snow depth changes are independent parameters, i.e., a systematic snow depth change does not produce a systematic intensity change. Figure 5, illustrating the scatter plot of the intensity change versus snow depth changes, shows no trend and indicates that the two parameters are independent.
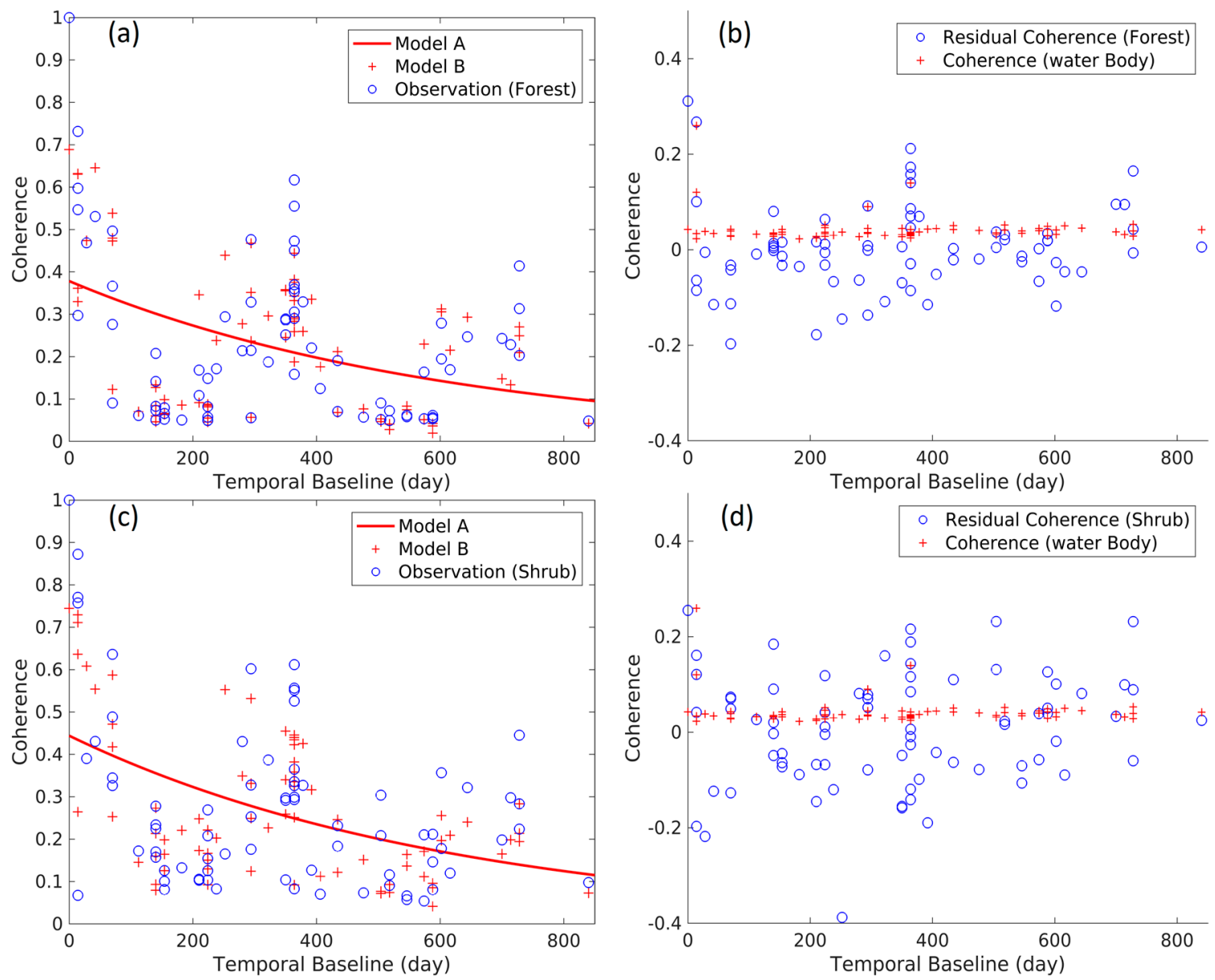

Figure 4. Scatter plots of observations and model B for forested (a) and shrub (c) land cover types. Scatter plots of residual coherences (observation-model B) for forested (b) and shrub (d) land cover types. Plot of model A and the scatter plot of coherence of water body are shown for comparison.

Table 2. Model parameters of the two land cover types. Cf is the critical $f$-value.

\begin{tabular}{ccccccccc}
\hline Model & Land Cover & $\gamma_{\mathbf{0}}$ & $\boldsymbol{\tau}$ (Day) & $\boldsymbol{\rho}$ & $\boldsymbol{\sigma}$ & RMS & $f$-Test & $\mathrm{C}_{\mathrm{f}}(\boldsymbol{\alpha}=\mathbf{0 . 0 1})$ \\
\hline \multirow{2}{*}{$\mathrm{A}$} & Forest & 0.37824 & 616.49 & - & - & 0.180 & - & - \\
& Shrub & 0.444 & 629.53 & - & - & 0.186 & - & - \\
\multirow{2}{*}{ B } & Forest & 0.68885 & 861.07 & 2.5406 & - & 0.092 & 205.84 & 6.99 \\
& Shrub & 0.74482 & 879.27 & 2.5467 & - & 0.121 & 102.38 & 6.99 \\
C & Forest & 0.73842 & 903.7 & 3.3464 & 0.62062 & 0.083 & 16.23 & 7.00 \\
& Shrub & 0.79153 & 913.47 & 5.6462 & 0.37348 & 0.101 & 29.64 & 7.00 \\
\hline
\end{tabular}



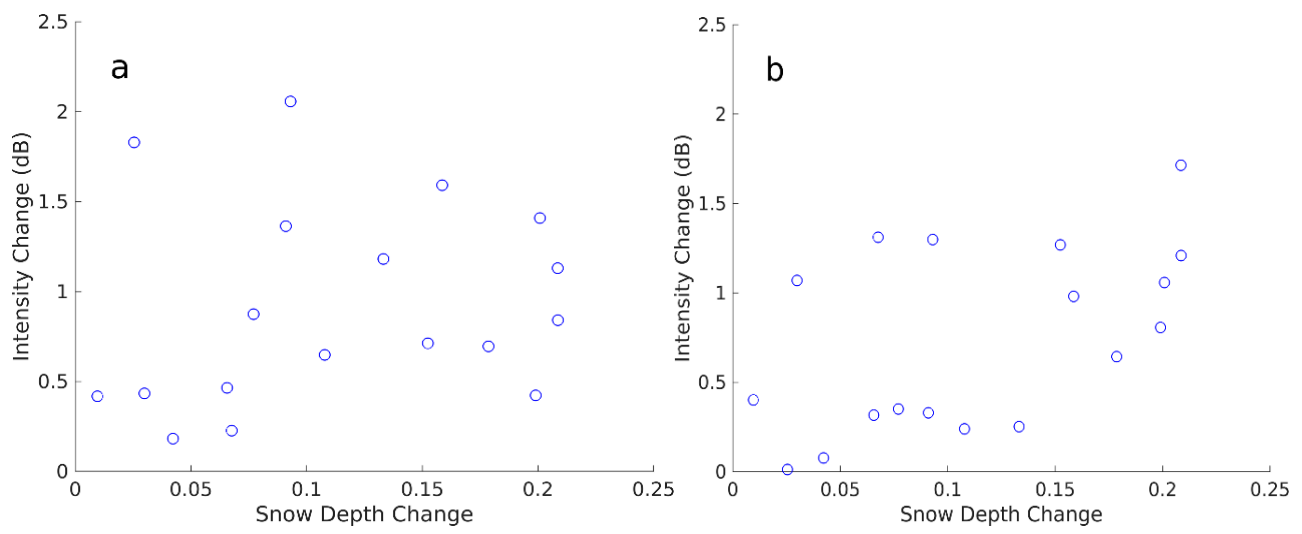

Figure 5. Scatter plot of model intensity changes $(\mathrm{dB})$ versus snow depth changes (meter) for forested (a) and shrub (b) land cover types.

Figure 6 shows semi-logarithmic scatter plot of coherence ratio, i.e., observation/model B, versus the snow depth change between two images. Considering the linear trend fitted to the semi-logarithmic scatter plots, model $\mathrm{C}$ is postulated to be:

$$
\gamma_{C}=\gamma_{0} e^{-\left(\frac{t}{\tau}+\frac{r}{\rho}+\frac{s}{\sigma}\right)}
$$

where $s$ is the snow depth change between images and $1 / \sigma$ is its decorrelation rate. The unknown parameters in model $C$ is estimated by solving the equations using known variables. The snow depth values are acquired from SMAP level 4 data (National Snow and Ice Data Center (http:/ /nsidc.org)) and the measurements at the SNOTEL Site Granite Creek (963) (Natural Water and Climate Center (https:/ /wcc.sc.egov.usda.gov)), which is located in our study area (white star in Figure 1). Figure 7 illustrates the scatter plots of model $C$, observed coherence, and residual coherence values (observation-model C) for the two landscapes. For comparison, the scatter plot of a water body's coherence is also shown. Additionally, Table 2 exhibits the model parameters and RMS error for each model. The RMS error values of model $C$ for the two land cover types are smaller than those of model B. This means that the change in snow depth leads to decorrelation and, taking into account its effect on InSAR coherence improves the model's performance. A statistical analysis of how significant the improvement is has been provided in the next section.
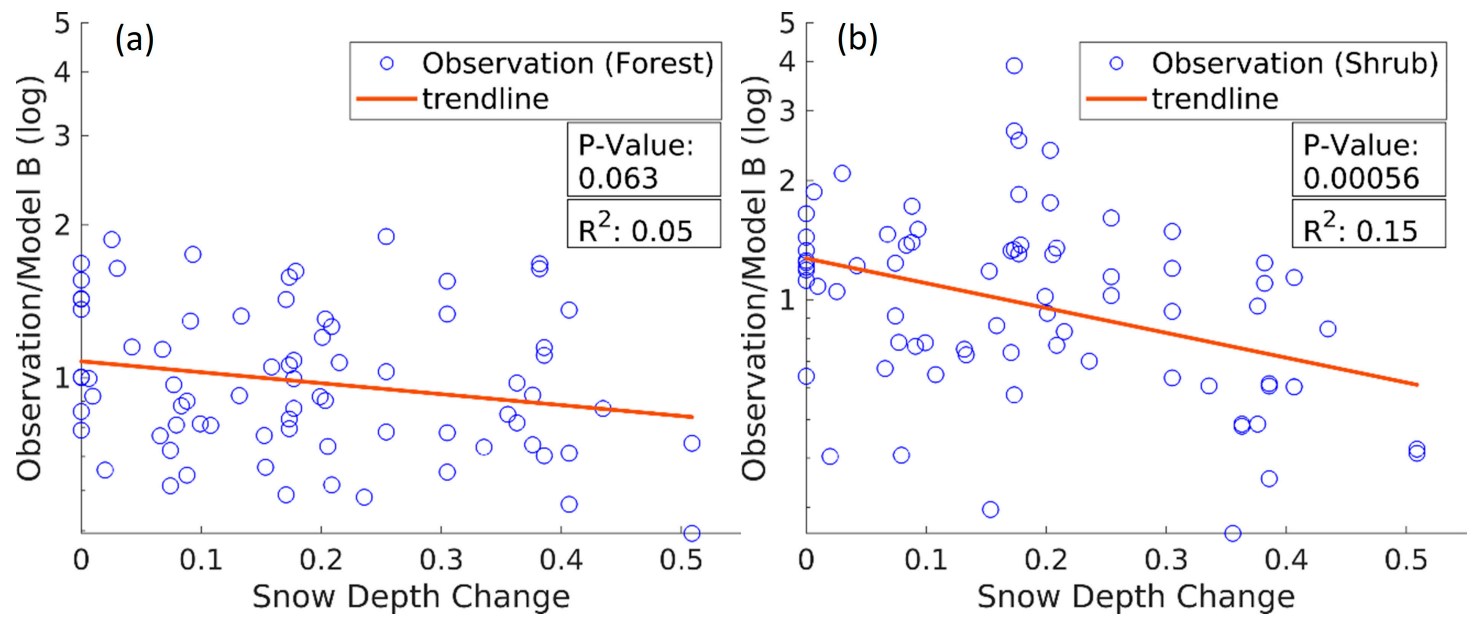

Figure 6. Semi-logarithmic scatter plot of the coherence ratio, i.e., observation/model B, versus snow depth changes (meter) for forested (a) and shrub (b) land cover types. 

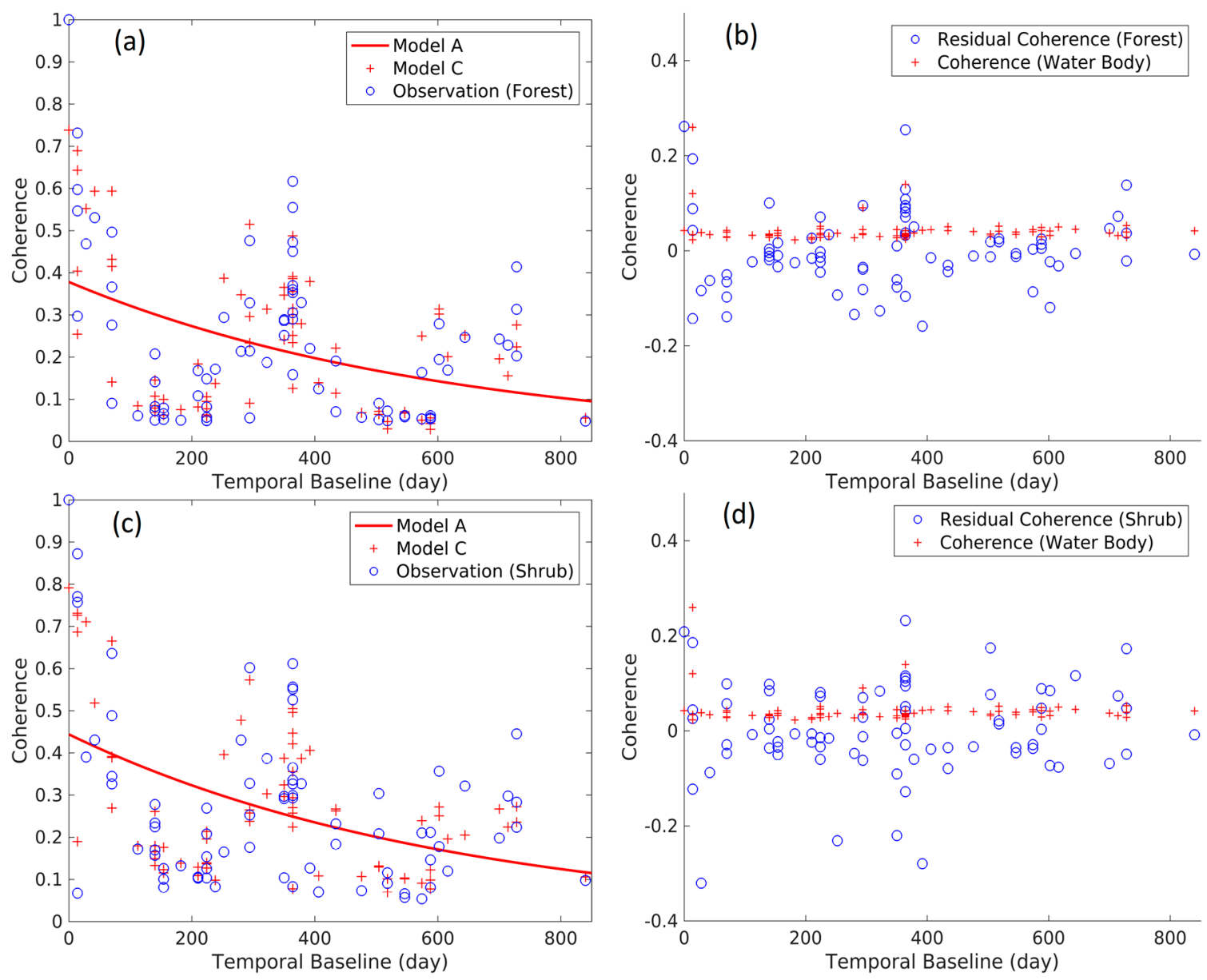

Figure 7. Scatter plots of observations and model C for forested (a) and shrub (c) land cover types. Scatter plots of residual coherences (observation-model C) for forested (b) and shrub (d) land cover types. Plot of model A and the scatter plot of coherence of water body are shown for comparison.

\section{Discussion}

\subsection{Scatterers' Type and Decorrelation Sources}

Model $C$ has three terms. The first term, $\exp (-t / \tau)$, is the long-term irreversible decorrelation due to the temporal change in scatterers' geometry. The second term, $\exp (-r / \rho)$, is the decorrelation due to the changes in backscattering between two images. As stated earlier, the change in SAR intensity was used as a proxy for the change in the surface backscattering. Figure 8 shows the plot of SAR intensity $(\mathrm{dB})$ over forest and shrub landscapes versus the soil moisture measured at the SNOTEL Site Granite Creek (963) (Natural Water and Climate Center (https: / /wcc.sc.egov.usda.gov)) which is located in our study area and is $12 \mathrm{~km}$ away on average from the patches (see Figure 1). The plots demonstrate a general correlation between soil moisture and SAR intensity, indicating that the change in backscattering and SAR intensity is most likely dominated by the change in dielectric constant of scatterers induced by the change in soil and biomass water content. 

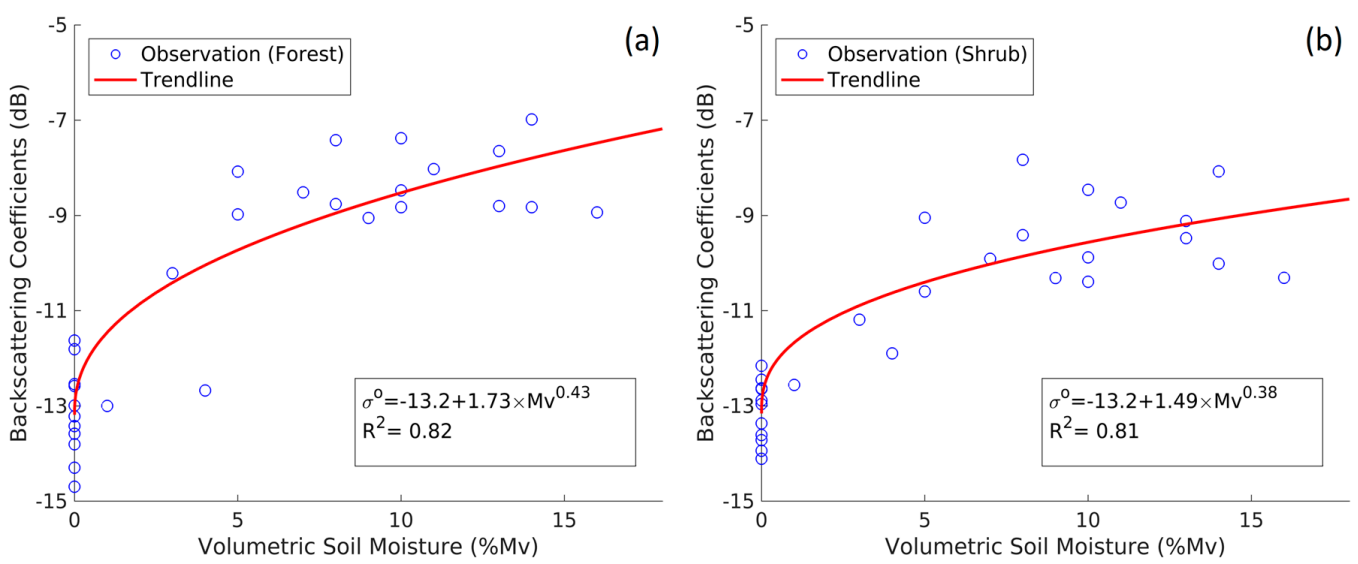

Figure 8. Plot of the change in SAR intensity for forested (a) and shrub (b) land cover types versus the change in soil moisture measured at the SNOTEL Site Granite Creek (963) (Natural Water and Climate Center (https: / / wcc.sc.egov.usda.gov)). Data under frozen and unfrozen conditions are included.

The third term, $\exp (-s / \sigma)$, is the decorrelation due to the change in snow depth between two images. The scatterplot of InSAR intensity change $(\mathrm{dB})$ versus snow depth change for soil moisture-free, i.e., winter-winter, interferograms (Figure 5) show no correlation between the SAR intensity change and the snow depth change over forested and shrub landscapes, indicating that snow depth change-induced decorrelation is most likely dominated by the change in the scatterers' geometry. In Section 4.3, we will perform a statistical assessment to show that the improvement made by considering the snow depth change in the model is statistically significant.

In the case where the temporal evolution of surface parameters such as soil moisture, vegetation layer parameters, and snow depth are known, the general coherence is postulated to be:

$$
\gamma=\gamma_{0} e^{-\left(\frac{t}{\tau}+\sum_{i=1}^{n} \frac{p_{i}}{\mu_{i}}\right)}
$$

where $1 / \mu_{i}$ is the decorrelation rate of the parameter $p_{i}$, which is the change in the surface parameters between two images.

In general, shrub landscape is more stable as changes happen in the scatterers' geometry and dielectric constant. Except the decorrelation rate of the change in snow depth, which is lower for forest, the decorrelation rates of long-term and backscattering are lower, i.e., higher $\tau$ and $\rho$ values, for shrub landscape compared to the forested landscape (Table 2). The scatterers within a resolution cell are of two types: scatterers associated with the ground surface and scatterers associated with the vegetation layer. Forested landscape (coniferous in this research) possess more backscattering contribution from the vegetation layer compared to the shrub landscape. Since the mutual position of scatterers within a vegetation layer, i.e., the geometry of scatterers, is more likely subject to change than the geometry of the scatterers within a non-vegetated surface, forested areas in the long-term decorrelate faster than non-forested areas as time lapses. Therefore, shrubland is expected to have a lower decorrelation rate associated with the long-term change in the scatterer's mutual position (Table 2). Additionally, the observed higher backscattering decorrelation rate of the forested area is associated, in part, with dielectric variation within the vegetation layer due to, for example, changing water content within the trees. Similarly, the observed higher snow depth decorrelation rate in shrubland is associated with the type of scatterers within each landscape. The lower proportion of snow-covered scatterers within the vegetation layer causes the forested landscape to lose coherence with a lower rate compared to the non-forested landscape as the change in snow depth increases.

The long-term decorrelation rate of the forest is slightly higher than the one of the shrub landscape, whereas the backscattering decorrelation rate of the forest is almost two times greater than that of the shrub landscape. This infers that, with short-baseline datasets, the difference between the temporal 
decorrelation of snow-free forested and non-forested areas is dominated by the decorrelation induced by the change in the dielectric constant of scatterers within the vegetation layer and not by the change in the geometry of scatterers within the vegetation layer.

\subsection{The Effect of Seasonality on Temporal Coherence}

Figure 9 depicts coherence as a 3D surface and provides a visual comparison of coherence evolutions of the two land cover types: forested and shrub. The x-axis of the plot is the relative change in SAR intensity and ranges between 0 and 0.65 , i.e., the maximum measured relative intensity change, whereas the $y$-axis is the change in snow depth and ranges between 0 and $0.65 \mathrm{~m}$. It is shown that both land cover types, even with the short temporal baselines, can lose coherence due to the changes in the dielectric constant and snow depth. Additionally, it is illustrated that the forest loses coherence with a higher rate than the shrub landscape with changing backscattering (dielectric constant), whereas the shrub landscape is more prone to decorrelation as the change in snow depth between two images increases.

Table 3 shows statistical properties of the observed coherence values of the two land cover types. The interferograms of each land cover type are subdivided into three sub-groups: summer, winter, and cross-season interferogram categories. It is shown that the shrub landscape has a higher coherence value than forest. Additionally, winter and cross-season interferogram categories possess the highest and lowest coherence values in general.
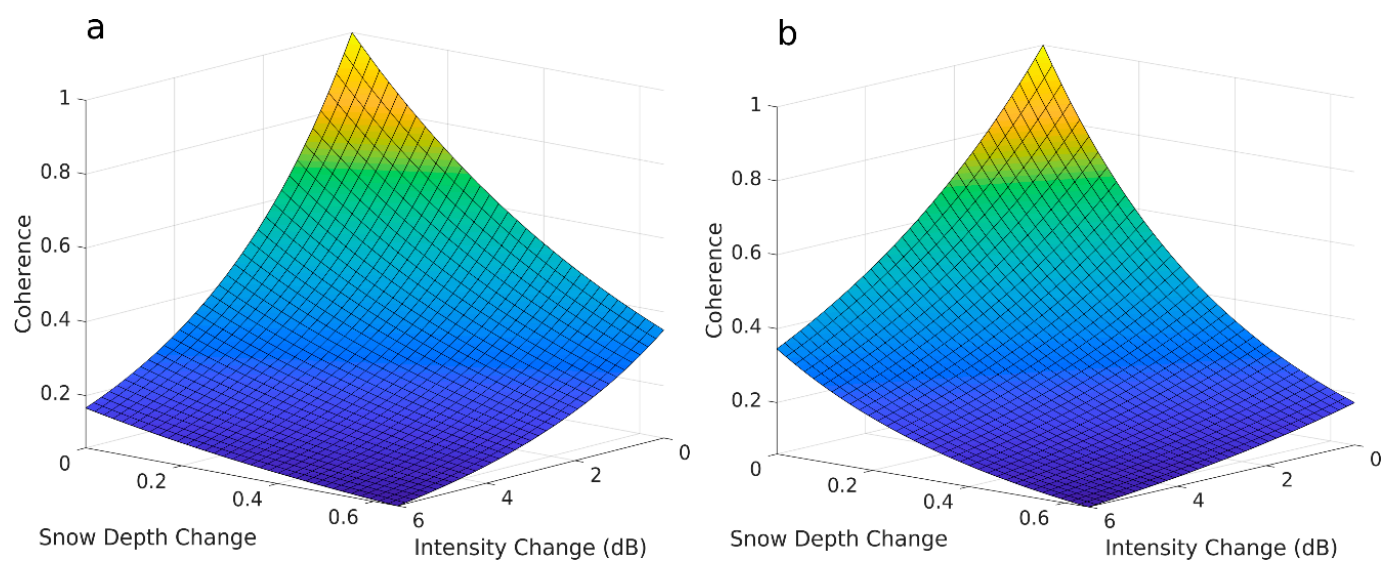

Figure 9. Coherence curves of forested (a); and shrub (b) land cover types.

Table 3. Statistical properties, i.e., mean and standard deviation (SD), of interferogram categories. The letters S, W, and C denote summer-summer, winter-winter, and cross-season interferogram groups.

\begin{tabular}{ccccc}
\hline Land Cover & Group & Mean & SD & SD/Mean \\
\hline \multirow{3}{*}{ Forest } & S & 0.3433 & 0.0711 & 0.2070 \\
& W & 0.3703 & 0.0680 & 0.1835 \\
& C & 0.0896 & 0.0407 & 0.4546 \\
\hline \multirow{3}{*}{ Shrub } & S & 0.3905 & 0.0974 & 0.2495 \\
& W & 0.4074 & 0.0836 & 0.2052 \\
& C & 0.1522 & 0.0566 & 0.3720 \\
\hline
\end{tabular}

Basically, the different scatterers' type and structure within the forested and non-forested resolution cells may result in different decorrelation processes when seasonal or sudden variations in surface parameters and meteorological conditions occur. During a frozen season, a decreased dielectric constant leads to reduced attenuation and a deeper penetration of electromagnetic waves into the forest canopy [34-36]. Consequently, this will cause a decrease in backscatter and influence the polarimetric signature and InSAR coherence [34-38]. In terms of coherence, between two winter images 
under stable frozen conditions, water content (soil moisture) changes do not occur, leading to low temporal decorrelation for open areas [34]. This means that higher InSAR coherence is expected for winter interferograms. Basically, over frozen forests, compared to the unfrozen condition, more volumetric decorrelation is expected to occur as the perpendicular baseline increases, owing to deeper penetration of electromagnetic waves into the frozen forest canopy [34]. However, with the short-baseline interferograms of L-band ALOS-2 and future datasets with narrower orbital tubes, the volumetric decorrelation induced by the deeper penetration of electromagnetic waves into the frozen soil and the frozen forest canopy become low, resulting in higher winter coherences.

During the unfrozen condition, changing soil moisture, variable water content within the trees, growth-related changes, and wind are among the major sources of temporal decorrelation $[39,40]$. The variation of the aforementioned surface parameters and meteorological conditions, in turn, results in decreased temporal coherence of the unfrozen condition compared to the frozen condition [34].

\subsection{Statistical Assessment on Models' Performance}

To statistically assess the improvements of models B and C, which have, respectively, one and two more parameters compared to model A, we used F-test (explained, for example, in Davis, 2002 [41]):

$$
F=\frac{S S R_{1}-S S R_{2}}{S S R_{2}} \frac{n-P_{2}}{P_{2}-P_{1}}
$$

where SSR is the sum of squared residuals of the model, $P$ is the number of free model parameters, and $n$ is the number of observations. If the calculated $F$-test value is greater than the upper-tailed critical value of the $F$-distribution, $\mathrm{F}_{\mathrm{P} 2-\mathrm{P} 1, \mathrm{n}-\mathrm{P} 2, \alpha}$, then, with a $1-\alpha$ percentage confidence, the null hypothesis is rejected, i.e., the improvement is statistically significant. The calculated $F$-test values and critical F-distribution values with 99\% confidence level (probability level of 0.01 ) have been presented in Table 2. All the calculated F-test values are greater than the critical values at a $99 \%$ confidence level, indicating that the improvements made by the new models are statistically significant.

\section{Conclusions}

Model C takes into account the long-term irreversible/long-term changes in scatterers' geometry, reversible/seasonal changes in scatterers' dielectric constant, induced mainly by the change in soil and biomass water content, and reversible/seasonal changes in scatterers' geometry, i.e., the third term, due to the change in snow depth. Shrub, in general is more stable than forest, as time lapses and variations happen in the surface backscattering properties. Additionally, the results show high coherence values for winter interferograms compared to summer ones owing to the stable condition of the frozen season. Our model illustrates that snow depth difference between interferogram pairs causes decorrelation, which is shown to mainly result from the change in the scatterers' geometry.

This paper argues that with short-baseline interferograms of L-band ALOS-2 and future data sets with narrower orbital tubes, the differences between temporal decorrelation rates of forest and non-forested areas, in snow-free condition, is dominated by the change in the dielectric constant of scatterers and not by the change in their geometry. It should be noted that the model introduced here is based on L-band data and, therefore, might not be expanded to C-band or X-band InSAR observations. The model provides accurate estimation of InSAR coherence for coniferous forested and shrub land cover types. However, its accuracy over other terrain types should be assessed.

Acknowledgments: ALOS-2 PALSAR data is copyrighted by the Japan Aerospace Exploration Agency (JAXA). We thank Paul Siqueira for facilitating the data access and Scott Hensley for providing several good references. The work was funded by the NASA Earth Surface and Interior Program (NNX16AK56G) and the Shuler-Foscue Endowment at Southern Methodist University. The authors would like to thank the academic editor and three reviewers for their very constructive comments and suggestions. 
Author Contributions: Yusuf Eshqi Molan processed the SAR data into In SAR products, conducted modeling, and created the first draft of the manuscript. Jin-Woo Kim, Zhong Lu, and Piyush Agram helped interpret the results. All contributed the writing of this manuscript. Readers who are interested in the data and products from this study can contact directly the corresponding author at the Southern Methodist University (yeshqimolan@smu.edu).

Conflicts of Interest: The authors declare no conflict of interest.

\section{References}

1. Massonnet, D.; Feigl, K. Radar interferometry and its application to changes in the Earth's surface. Rev. Geophys. 1998, 36, 441-500. [CrossRef]

2. Bürgmann, R.; Rosen, P.A.; Fielding, E.J. Synthetic Aperture Radar Interferometry to Measure Earth's Surface Topography and Its Deformation. Annu. Rev. Earth Planet. Sci. 2000, 28, 169-209. [CrossRef]

3. Simons, M.; Rosen, P. Treatise on Geophysics: Interferometric Synthetic Aperture Radar Geodesy. In Geodesy; Schubert, G., Ed.; Elsevier Press: Amsterdam, The Netherlands, 2007; Volume 3, pp. 391-446.

4. Lu, Z.; Dzurisin, D. InSAR imaging of Aleutian volcanoes: Monitoring a volcanic arc from space. In Geophysical Sciences; Springer Praxis Books: Chichester, UK, 2014; 390p.

5. Ferretti, A.; Prati, C.; Rocca, F. Permanent scatterers in SAR interferometry. IEEE Trans. Geosci. Remote Sens. 2001, 39, 8-20. [CrossRef]

6. Kim, J.W.; Lu, Z.; Degrandpre, K. Ongoing Deformation of Sinkholes in Wink, Texas, Observed by Time-Series Sentinel-1A SAR Interferometry (Preliminary Results). Remote Sens. 2016, 8, 313. [CrossRef]

7. Hu, X.; Wang, T.; Pierson, T.C.; Lu, Z.; Kim, J.; Cecere, T.H. Detecting seasonal landslide movement within the Cascade landslide complex (Washington) using time-series SAR imagery. Remote Sens. Environ. 2016, 187, 49-61. [CrossRef]

8. Rykhus, R.; Lu, Z. InSAR detects possible thaw settlement in the Alaskan Arctic Coastal Plain. Can. J. Remote Sens. 2008, 34, 100-112. [CrossRef]

9. Liu, L.; Zhang, T.; Wahr, J. InSAR measurements of surface deformation over permafrost on the North Slope of Alaska. J. Geophys. Res. 2010, 115, F03023. [CrossRef]

10. Zebker, H.; Villasenor, J. Decorrelation in interferometric radar echoes. IEEE Trans. Geosci. Remote Sens. 1992, 45, 950-959. [CrossRef]

11. Just, D.; Bamler, R. Phase statistics of interferograms with applications to synthetic aperture radar. Appl. Opt. 1994, 33, 4361-4368. [CrossRef] [PubMed]

12. Simard, M.; Hensley, S.; Lavalle, M. An empirical assessment of temporal decorrelation using the uninhabited aerial vehicle synthetic aperture radar over forested landscapes. Remote Sens. 2012, 4, 975-986. [CrossRef]

13. Rocca, F. Modeling Interferograms Stacks. IEEE Trans. Geosci. Remote Sens. 2007, 45, 3289-3299. [CrossRef]

14. Luo, X.; Askne, J.; Smith, G.; Dammert, P. Coherence characteristics of RADAR signals from rough soil. Prog. Electromagn. Res. 2001, 31, 68-88. [CrossRef]

15. Zwieback, S.; Hensley, S.; Hajnsek, I. Assessment of soil moisture effects on L-band radar interferometry. Remote Sens. Environ. 2015, 164, 77-89. [CrossRef]

16. Zwieback, S.; Paulik, C.; Wagner, W. Frozen Soil Detection Based on Advanced Scatterometer Observations and Air Temperature Data as Part of Soil Moisture Retrieval. Remote Sens. 2015, 7, 3206-3231. [CrossRef]

17. Zwieback, S.; Hensley, S.; Hajnsek, I. A Polarimetric First-Order Model of Soil Moisture Effects on the DInSAR Coherence. Remote Sens. 2015, 7, 7571-7596. [CrossRef]

18. Lavalle, M.; Simard, M.; Hensley, S. A Temporal Decorrelation Model for Polarimetric Radar Interferometers. IEEE Trans. Geosci. Remote Sens. 2012, 50, 2880-2888. [CrossRef]

19. Zhang, L.; Shi, J.; Zhang, Z.; Zhao, K. The estimation of dielectric constant of frozen soil-water mixture at microwave bands. In Proceedings of the IEEE International Geoscience and Remote Sensing Symposium, Toulouse, France, 21-25 July 2003; IEEE: Piscataway, NJ, USA, 2003; pp. 2903-2905.

20. Zhang, T.; Zeng, Q.; Li, Y.; Xiang, Y. Study on relation between InSAR coherence and soil moisture. In Proceedings of the ISPRS Congress, Beijing, China, 3-11 July 2008.

21. Borgeaud, M.; Wegmueller, U. On the use of ERS SAR interferometry for the retrieval of geoand bio-physical information. In Proceedings of the 'Fringe 96' Workshop on ERS SAR Interferometry, Zurich, Switzerland, 30 September-2 October 1996; pp. 83-94. 
22. Morishita, Y.; Hanssen, R.F. Temporal Decorrelation in L-, C-, and X-band Satellite Radar Interferometry for Pasture on Drained Peat Soils. IEEE Trans. Geosci. Remote Sens. 2015, 53, 1096-1104. [CrossRef]

23. Lee, S.-K.; Kugler, F.; Papathanassiou, K.P.; Hajnsek, I. Quantification of Temporal Decorrelation Effects at L-Band for Polarimetric SAR Interferometry Applications. IEEE J. Sel. Top. Appl. Earth Obs. Remote Sens. 2013, 6, 1351-1367. [CrossRef]

24. Homer, C.G.; Dewitz, J.A.; Yang, L.; Jin, S.; Danielson, P.; Xian, G.; Coulston, J.; Herold, N.D.; Wickham, J.D.; Megown, K. Completion of the 2011 National Land Cover Database for the conterminous United StatesRepresenting a decade of land cover change information. Photogramm. Eng. Remote Sens. 2015, 81, 345-354.

25. O'Neill, K.P.; Kasischke, E.S.; Richter, D.D. Seasonal and decadal patterns of soil carbon uptake and emission along an age-sequence of burned black spruce stands in interior Alaska. J. Geophys. Res. 2003, 108, 11-15. [CrossRef]

26. Lu, Z.; Freymueller, J. Synthetic aperture radar interferometry coherence analysis over Katmai volcano group, Alaska. J. Geophys. Res. 1998, 103, 29887-29894. [CrossRef]

27. Dzurisin, D.; Lu, Z. Interferometric Synthetic Aperture Radar (InSAR) (Chapter 5): Volcano Deformation: Geodetic Monitoring Techniques; Dzurisin, D., Ed.; Springer-Praxis Publishing Ltd.: Chichester, UK, 2007; pp. 153-194.

28. Hanssen, R. Radar Interferometry: Data Interpretation and Error Analysis; Kluwer: Dordrecht, The Netherlands, 2001; Volume 2.

29. Bamler, R.; Hartl, P. Synthetic aperture radar interferometry. Inverse Prob. 1998, 14, R1-R54. [CrossRef]

30. Touzi, R.; Lopes, A.; Bruniquel, J.; Vachon, P. Coherence estimation for SAR imagery. IEEE Trans. Geosci. Remote Sens. 1999, 37, 135-149. [CrossRef]

31. Lombardini, F.; Griffiths, H. Effect of temporal decorrelation on 3D SAR imaging using multiple pass beamforming. In Proceedings of the IEE/EUREL Meeting Radar Sonar Signal Processing, Peebles, UK, 5-8 July 1998; pp. 1-4.

32. Ulaby, F.T.; Bradley, G.A.; Dobson, M.C. Microwave backscatter dependence on surface roughness, soil moisture, and soil texture, II, Vegetation covered soil. IEEE Trans. Geosci. Electron. 1979, 17, 33-40. [CrossRef]

33. Zribi, M.; Gorrab, A.; Baghdadi, N. A new soil roughness parameter for the modelling of radar backscattering over bare soil. Remote Sens. Environ. 2014, 152, 62-73. [CrossRef]

34. Thiel, C.; Schmullius, C. The potential of ALOS PALSAR backscatter and InSAR coherence for forest growing stock volume estimation in Central Siberia. Remote Sens. Environ. 2016, 173, 258-273. [CrossRef]

35. Kwok, R.; Rignot, E.J.M.; Way, J.; Freeman, A.; Holt, J. Polarization signatures of frozen and thawed forests of varying environmental state. IEEE Trans. Geosci. Remote Sens. 1994, 32, 371-381. [CrossRef]

36. Way, J.; Paris, J.; Kasischke, E.; Slaughter, C.; Viereck, L.; Christensen, N.; Weber, J. The effect of changing environmental-conditions on microwave signatures of forest ecosystems-Preliminary-results of the March 1988 Alaskan aircraft SAR experiment. Int. J. Remote Sens. 1990, 11, 1119-1144. [CrossRef]

37. Dobson, M.G.; McDonald, K.; Ulaby, F.T. Effects of temperature on radar backscatter from boreal forests. In Proceedings of the 10th Annual International Geoscience and Remote Sensing Symposium, College Park, MD, USA, 20-24 May 1990; Mills, R., Ed.; IEEE Publications: College Park, MD, USA, 1990; pp. 2481-2484.

38. Santoro, M.; Eriksson, L.; Fransson, J. Reviewing ALOS PALSAR backscatter observations for stem volume retrieval in Swedish forest. Remote Sens. 2015, 7, 4290-4317. [CrossRef]

39. Dobson, M.C. Diurnal and seasonal variations in the microwave dielectric constant of selected trees. In Proceedings of the International on Geoscience and Remote Sensing Symposium, Edinburgh, UK, 12-16 September 1988; Guyenne, T.D., Hunt, J.J., Eds.; ESA Publications Devision: Edinburgh, UK, 1988; p. 1754.

40. McDonald, K.C.; Zimmermann, R.; Kimball, J.S. Diurnal and spatial variation of xylem dielectric constant in Norway spruce (Picea abies [L.] karst.) as related to microclimate, xylem sap flow, and xylem chemistry. IEEE Trans. Geosci. Remote Sens. 2002, 40, 2063-2082. [CrossRef]

41. Davis, J.C. Statistics and Data Analysis in Geology, 3rd ed.; John Wiley and Sons: Hoboken, NJ, USA, 2002.

(C) 2018 by the authors. Licensee MDPI, Basel, Switzerland. This article is an open access article distributed under the terms and conditions of the Creative Commons Attribution (CC BY) license (http:/ / creativecommons.org/licenses/by/4.0/). 